\title{
Competitividade e efeito de medidas políticas no consórcio cacaueiro-seringueira
}

\author{
Competitiveness and effect of policy measures in production systems with cocoa \\ and rubber tree
}

Adrielli Santos de Santana ${ }^{1}$ [D, Naisy Silva Soares² (D), Moema Maria Badaró Cartibani Midlej² (D), Adonias Castro Virgens Filho ${ }^{3}$

${ }^{1}$ Universidade de Brasília (UnB), Brasília (DF), Brasil. E-mail: adrielli_santana@outlook.com

2Universidade Estadual de Santa Cruz (UESC), Ilhéus (BA), Brasil. E-mails: naisysilva@yahoo.com.br; moema.midlej@gmail.com

${ }^{3}$ Comissão Executiva do Plano da Lavoura Cacaueira (CEPLAC), Ilhéus (BA), Brasil. E-mail: adoniascastro@uol.com.br

Como citar: Santana, A. S., Soares, N. S., Midlej, M. M. B. C., \& Virgens Filho, A. C. (2020). Competitividade e efeito de medidas políticas no consórcio cacaueiro-seringueira. Revista de Economia e Sociologia Rural, 58(3), e191906.

https://doi.org/10.1590/1806-9479.2020.191906

Resumo: O presente trabalho analisou a competitividade e a eficiência do sistema de produção consorciada do cacaueiro com a seringueira, no sul do Estado da Bahia, através do método da Matriz de Análise de Políticas desenvolvida por Monke e Pearson. Foram mensurados os indicadores da MAP, agrupando-os em quatro categorias: análise de lucratividade; análise de eficiência; análise de proteção; e transferência líquida de políticas. Os resultados obtidos permitem afirmar que a consorciação das culturas do cacau com a seringueira é uma atividade lucrativa e competitiva, do ponto de vista privado e social. Todavia, os produtores são penalizados pela presença de políticas que incidem sobre o produto e sobre os insumos. Além disso observou-se que o comportamento dos indicadores está condicionado às oscilações nos preços do cacau e da borracha natural.

Palavras-chave: matriz de análise política, cacau, heveicultura, análise de risco.

Abstract: The present work analyzed the competitiveness and efficiency of the consortium production system of the cocoa with the rubber tree, in the southern Bahia, through the policies analysis matrix method developed by Monke and Pearson. PAM indicators were measured and grouped into four categories: profitability analysis; efficiency analysis; protection analysis; and net policies transfers. The obtained results allow affirming that the intercropping of cocoa crops with the rubber tree is a lucrative and competitive activity, from a private and social point of view. However, producers are penalized by policies that affect the product and inputs. Moreover, it was observed that indicators' behavior are conditioned to cocoa and natural rubber prices fluctuations.

Keywords: policy analysis matrix, cocoa, natural rubber, risk analysis.

\section{INTRODUÇÃO}

Os estudos voltados para a análise e avaliação da competitividade em sistemas de agronegócios são de suma importância para o desenvolvimento das atividades deste setor. Entre os métodos desenvolvidos para mensurar a competitividade, a Matriz de Análise Política (MAP), do original, em inglês, Policy Analysis Matrix (PAM), elaborada em 1981 por Eric A. Monke e Scott R. Pearson demonstra os impactos de políticas sobre os custos e a lucratividade de um sistema agrícola, avaliados por meio da comparação entre os cenários com e sem a presença de políticas (Monke \& Pearson, 1989).

A aplicação da MAP para análises de sistemas agrícolas e de agronegócios não é novidade dentro das literaturas especializadas nesta temática. Desde a sua criação, surgiram diversos estudos que visavam demonstrar as mudanças comportamentais em diferentes sistemas agrícolas em diversos países. Na literatura internacional, por exemplo, destacam-se 
os trabalhos de Adesina \& Coulibaly (1998), para a produção de milho em Camarões, Fang e Beghin (2000), para a agricultura chinesa; Mohanty et al. (2002), para a cotocicultura indiana; Pearson et al. (2003), para a agricultura da Indonésia; Kanaka \& Chinnadurai (2015) para o cultivo de arroz na Índia, entre muitos outros.

De forma semelhante, ocorre na literatura nacional. Segundo Freitas (2013, p. 26), os estudos de competitividade em sistemas produtivos e cadeias produtivas, utilizando a MAP, se restringiram a alguns órgãos governamentais e institutos de pesquisas, a exemplo da "Empresa Brasileira de Pesquisa Agropecuária (Embrapa), Fundação Getúlio Vargas (FGV) e algumas universidades". Estes órgãos foram responsáveis pela aplicação do método para os principais produtos agrícolas produzidos no território brasileiro (algodão, arroz, café, feijão, leite, milho, soja, trigo, cacau).

Entretanto, este fato não impediu o desenvolvimento de estudos complementares. Entre eles, têm-se os trabalhos de Pires (1996), para avaliar a expansão da produção de arroz, milho, feijão e soja no Estado de Minas Gerais; Milhomem \& Teixeira (2001), para os principais estados brasileiros produtores de café; Alvim et al. (2004), para a produção de soja na região do cerrado brasileiro; Ferreira Neto (2005), para a monocultura da cana-de-açúcar; Moss (2006), para o cultivo de café arábica em Minas Gerais e São Paulo; Soares et al. (2010), para a cadeira produtiva da madeira de eucalipto; Sousa et al. (2011), para a produção de biodiesel de dendê no Baixo Sul da Bahia; Freitas (2013), para a produção de leite em pó, e Pacheco (2014), para a cadeia produtiva da maçã, ambos no Rio Grande do Sul; e Araújo (2015), para a cadeia produtiva da manga em Pernambuco, entre muitos outros.

Observa-se que a aplicação do método da MAP contemplou uma grande parcela dos produtos agrícolas nacionais. Entretanto, as alternativas de pesquisas não foram totalmente esgotadas. Diante desse contexto, o presente trabalho tem como objetivo geral analisar a competitividade e o efeito de políticas públicas sobre os sistemas de produção consorciada do cacau com a seringueira, no sul da Bahia. Especificamente, buscou-se: determinar a lucratividade privada e social do consórcio; mensurar os indicadores que avaliam o grau de competitividade, a eficiência econômica e os efeitos das políticas nestas atividades, levando em consideração os resultados privados e sociais; e analisar a sensibilidade dos indicadores privados e sociais devida a uma variação na taxa de câmbio.

Salienta-se, que a competitividade da cadeia produtiva do cacau foi analisada por meio deste método, em estudo desenvolvido pela Embrapa e por Vieira et al. (2001). De modo semelhante, o estudo foi realizado, por Rosado et al. (2006), para o sistema de produção da borracha natural. Todavia, pretende-se aqui atualizar a literatura existente, demonstrar as novas configurações dessas culturas por meio de consorciações e inserir o debate sobre o papel das políticas públicas para o desenvolvimento dessas atividades na região.

Observa-se que a produção de cacau e de borracha natural continuam a desempenhar um papel significativo na economia do Estado, sobretudo sobre as receitas de exportações de produtos agroindustriais. Conforme dados divulgados pela Superintendência de Estudos Econômicos e Sociais da Bahia (2017), no ano de 2015, foram exportados um volume de 268.676 toneladas de cacau e derivados, equivalendo a um montante de 62,2 milhões de dólares (US\$ FOB), e 211.455 toneladas de borrachas e suas obras, equivalentes a 45,08 milhões de dólares (US\$FOB), valores superados pelas exportações de soja, de algodão, de frutas e suas preparações, e de sisal, juntamente com seus respectivos subprodutos.

A economia cacaueira vivencia, na conjuntura atual, um processo de recuperação pós-crise, marcado pelo desenvolvimento sucessivo de estratégias para retomar a produtividade, a qualidade e a competitividade da produção regional. Dentre as estratégias de recuperação, a consorciação de culturas possui diversas vantagens, dentre as quais ressaltam o maior aproveitamento das áreas plantáveis, a diminuição no dispêndio de insumos produtivos, a redução nos custos de produção e, consequentemente, a geração de renda, principalmente, para os pequenos produtores que se beneficiam com os ganhos na produção de duas ou mais culturas.

O cacau, em algumas regiões, já é produzido de forma consorciada com outras espécies, a exemplo da banana, coco, pimenta-do-reino e a seringueira. Dentre estes produtos, a seringueira se destaca por fornecer a matéria-prima - o látex - para a produção de borracha natural, que por sua vez é uma importante commodity agrícola brasileira, assim como o cacau, 
sendo o Estado da Bahia o segundo maior produtor nacional. Além disso, a heveicultura desempenha um importante papel ambiental ao contribuir para o reflorestamento de áreas degradadas.

Nessa perspectiva, a presente pesquisa pauta-se na seguinte questão norteadora: quão competitivos são os sistemas de produção consorciado do cacau com a seringueira no sul do Estado da Bahia?

A resposta a esta indagação possui grande relevância entre os estudos que visam identificar os aspectos determinantes da competitividade da cacauicultura baiana, assim como da heveicultura. Concomitantemente, identificar as fragilidades nas suas estruturas de custos, objetivando a manutenção e/ou a ampliação da capacidade produtiva e da lucratividade da produção.

\section{METODOLOGIA}

\subsection{Matriz de Análise Política}

A MAP tem sido, constantemente, aplicada para estimar os lucros de diversos sistemas agrícolas e os impactos sociais gerados por eles, considerando as variações nos cenários produtivos, tecnológicos e institucionais (Kanaka \& Chinnadurai, 2015).

Em síntese, este método tem por finalidade fornecer uma visão delineada das relações entre elos de cadeias produtivas, ou sistemas produtivos individuais, ressaltando suas relações de interdependência setorial e seu desempenho econômico, através da identificação das fragilidades no sistema de custo e na aplicação eficiente dos recursos, além de possibilitar a avaliação dos impactos econômicos gerados pela adoção de políticas sobre os sistemas agrícolas (Ferreira Neto, 2005; Pacheco, 2014; Araújo, 2015).

Os objetivos da MAP podem ser pautados sobre três questões norteadoras que auxiliam os formuladores de políticas públicas no processo de avaliação de impactos de políticas, sendo elas: (i) a competitividade do sistema agrícola, em função dos preços praticados no mercado; (ii) os efeitos de investimentos públicos em infraestrutura sobre a eficiência do sistema produtivo; e (iii) os ganhos de eficiência gerados através de investimentos públicos em pesquisas e tecnologias voltadas à atividade agrícola (Monke \& Pearson, 1989; Pearson et al., 2003; Araújo, 2015).

Conforme afirmado por Moss (2006), o desempenho dos sistemas agrícolas tende a ser muito sensível aos efeitos de políticas públicas. Nestas condições, as políticas geradas a partir destes três pontos influem diretamente sobre o valor de produção e, consequentemente, as receitas auferidas pelos produtos, os custos dos insumos e os lucros dos sistemas agrícolas. Portanto, permitindo estimar a competitividade, a lucratividade privada e social, sendo estes analisados em termos de eficiência, e, por fim, os efeitos de transferências de políticas, sendo estes os objetivos principais do uso da MAP (Monke \& Pearson, 1989; Pearson et al., 2003; Araújo, 2015).

Entre as vantagens da utilização do método MAP, está o seu baixo custo de manutenção, visto que, após o levantamento dos dados empíricos e a mensuração dos seus coeficientes para determinado período-base, o acompanhamento do desempenho agrícola e os efeitos de políticas, para os períodos subsequentes, podem ser, frequentemente, atualizados, permitindo um maior controle sobre os entraves nas estruturas de custos e na lucratividade (Pacheco, 2014). Todavia, ressalta-se que, apesar de ser um instrumento simples, ele possui um rigoroso suporte teórico-analítico baseado nas teorias de custo-benefício social e de comércio internacional em economia (Kanaka \& Chinnadurai, 2015).

\subsection{Operacionalização da MAP}

A construção empírica da Matriz de Análise de Políticas é resultante do produto de duas identidades contábeis: (i) a lucratividade, que é definida como a diferença entre as receitas e os custos privados; e (ii) os efeitos das divergências, associados às distorções de políticas e/ou falhas de mercado, observados por meio da diferença entre os preços privados, praticados 
no mercado, e os preços sociais, que representam os custos de oportunidade (Monke \& Pearson, 1989; Araújo, 2015; Pacheco, 2014).

A MAP fornece dados sobre as receitas, custos e lucros do sistema agrícola. Assim, o Quadro 1 ilustra a construção da MAP e dos respectivos elementos que a constituem.

Quadro 1 - Matriz de análise política simplificada.

\begin{tabular}{|c|c|c|c|c|} 
& \multicolumn{4}{c}{ Custos } \\
\cline { 3 - 5 } Discriminação & Receitas & $\begin{array}{c}\text { Insumos } \\
\text { comercializáveis }\end{array}$ & $\begin{array}{c}\text { Fatores } \\
\text { domésticos }\end{array}$ \\
\hline Preços privados & & $\mathrm{B}$ & $\mathrm{C}$ & $\mathrm{D}$ \\
\hline Preços sociais & $\mathrm{A}$ & $\mathrm{F}$ & $\mathrm{G}$ & $\mathrm{H}$ \\
\hline $\begin{array}{c}\text { Efeitos de divergência e eficiência } \\
\text { política }\end{array}$ & $\mathrm{I}$ & $\mathrm{J}$ & $\mathrm{K}$ & $\mathrm{L}$ \\
\hline
\end{tabular}

Fonte: Monke \& Pearson (1989).

A primeira linha da MAP contém os preços privados do sistema agrícola, onde: A representa as receitas privadas; $B$ mede os custos dos fatores de produção comercializáveis; C representa os custos de fatores domésticos; e D é lucro privado (LP), resultante da diferença das receitas e dos custos privados. O cálculo do lucro privado pode ser descrito pela seguinte expressão:

Dou LP $=\mathrm{A}-(\mathrm{B}+\mathrm{C})$

Dentro do método da MAP, a medição do lucro privado é um importante sinalizador de competitividade do sistema agrícola. A análise é baseada na capacidade de as receitas privadas suprirem os custos do capital e os custos de oportunidade englobados aos fatores domésticos. Desta forma, o cálculo do lucro privado pode assumir um dos três possíveis resultados: (i) lucros privados negativos $(\mathrm{LP}<0)$ indicam que o sistema agrícola está sendo operado a uma taxa de retorno subnormal, sendo necessária uma intervenção para aumentar os lucros ou o encerramento das atividades de produção; (ii) lucro normal $(\mathrm{LP}=0)$ sinaliza que as receitas privadas geradas pelo sistema equivalem ao valor dos custos privados, logo os operadores não detêm nem lucros nem prejuízos; e, por fim, (iii) lucros privados positivos (LP >0) indicam a existência de taxas de retornos sobrenormais, ou seja, os operadores estão auferindo lucros com o sistema agrícola, e este cenário indica uma possível expansão produtiva, em uma perspectiva futura, mantidos todos os fatores (endógenos e exógenos) favoráveis.

A mesma analogia, realizada anteriormente, serve para a segunda linha da matriz, entretanto esta representa os preços sociais, que podem ser entendidos como os valores resultantes da alocação eficiente dos recursos. Desta forma, os símbolos contidos nesta linha podem ser lidos da seguinte maneira: E mede as receitas a preços sociais; $F$ são os custos de insumos negociáveis em preços sociais; $G$ representa o fator doméstico em preços sociais; e H é lucro social (LS), obtido da expressão:

Hou LS $=\mathrm{E}-(\mathrm{F}+\mathrm{G})$

Os lucros sociais indicam a eficiência ou sua vantagem comparativa do sistema agrícola em análise. A interpretação do lucro social permite verificar se a aplicação dos recursos proporciona um nível ótimo de produção e de renda. Nesse contexto, o lucro social pode apresentar valores negativos ( $\mathrm{LS}<0)$, nulos $(\mathrm{LS}=0)$, ou positivos $(\mathrm{LS}>0)$. No primeiro caso, lucros sociais negativos implicam a ineficiência do sistema agrícola quanto à aplicação dos recursos, tornando-o inviável em termos econômicos, e supondo a necessidade de intervenção governamental para a manutenção das atividades. O segundo caso indica a 
ocorrência de lucros normais, semelhante à interpretação obtida considerando os preços privados. No terceiro e último caso, os lucros sociais positivos indicam que o sistema agrícola é eficiente e economicamente viável.

A terceira linha da MAP corresponde aos efeitos de divergência e eficiência política, obtidos pela subtração das parcelas sociais nos componentes privados de receitas, custos e lucros. Em outras palavras, representa as transferências do governo, através dos efeitos de todas as políticas públicas consideradas no modelo, onde: I mede o volume de transferências associadas à produção $(\mathrm{A}-\mathrm{E})$; J indica as transferências aos custos dos insumos $(\mathrm{B}-\mathrm{F})$; $\mathrm{K}$ mede as transferências aos custos dos fatores $(\mathrm{C}-\mathrm{G})$; e $\mathrm{L}$ indica as transferências líquidas de políticas $(\mathrm{D}-\mathrm{H})$, podendo ser escrita pela seguinte expressão:

$\operatorname{Lou} \mathrm{TLP}=\mathrm{I}-(\mathrm{J}+\mathrm{K})$

Cujos resultados indicam que o governo transfere renda ao sistema agrícola, quando o montante de transferências líquidas é positivo (TLP $>0$ ), podendo, também, extrair a renda do sistema agrícola para a sociedade, quando as transferências líquidas apresentam resultados negativos (TLP $<0$ ), ou nenhuma das opções anteriores, sendo, portanto, neutro (TLP $=0$ ) .

Além de mensurar os valores de LP, LS e do TLP, os dados dispostos no formato da MAP permitem estimar outros indicadores (Monke \& Pearson, 1989; Freitas, 2013; Ferreira Neto, 2005; Moss, 2006; Alvim et al., 2004; Araújo, 2015; Pearson et al., 2003;), apresentados a seguir:

a) Razão do Custo Privado (RCP) - indica a lucratividade do sistema agrícola, demonstrando o montante de renda a preços privados que ele é capaz de gerar para cobrir os custos dos fatores domésticos e, ainda, se manter competitivo no mercado, após obter lucros normais (Alvim et al., 2004). O RCP pode ser descrito pela seguinte equação:

$\mathrm{RCP}=\frac{\mathrm{C}}{(\mathrm{A}-\mathrm{B})}$

Onde: $\mathrm{RCP}=1$ demonstra a equidade da remuneração dos fatores domésticos a retornos normais; $\mathrm{RCP}<1$ indica que os fatores domésticos estão recebendo mais do que seu retorno normal, portanto o sistema agrícola se mantém competitivo; e RCP $>1$, o sistema não é capaz de remunerar os fatores domésticos, logo, estes recebem abaixo do retorno normal.

b) Razão de custo dos insumos domésticos (RCD) - indica a eficiência ou a vantagem comparativa do sistema agrícola, sendo mais preciso que o cálculo do LS. Este indicador permite visualizar a lucratividade social, ou seja, a remuneração dos fatores domésticos, a preços sociais, em função do custo de oportunidade. A equação matemática para a RCD é indicada por:

$\mathrm{RCD}=\frac{\mathrm{G}}{(\mathrm{E}-\mathrm{F})}$

Cujos resultados apontam que: $\mathrm{RCD}=1$, a remuneração dos fatores domésticos, a preços sociais, equivale ao custo de oportunidade do sistema agrícola; $\mathrm{RCD}<1$ indica que os fatores domésticos estão recebendo mais do que seu custo de oportunidade, portanto apresenta lucros positivos; e RCD $>1$, os fatores domésticos sociais não são remunerados, e o sistema agrícola apresenta lucros negativos.

c) Coeficiente de proteção nominal (CPN) - expressa a razão entre os preços privados e o preço social, demonstrando os efeitos das políticas de governo, ou das falhas existentes no mercado. Sinaliza, pois, as transferências de renda entre os produtores e a sociedade, sendo expresso por:

$\mathrm{CPN}=\frac{\mathrm{A}}{\mathrm{E}}$ 
A não transferência de renda dentro do sistema agrícola ocorre quando o CPN assume valor igual a 1. Para $\mathrm{CPN}<1$, a interpretação dada é a de que os produtores recebem valores menores que o preço de mercado, portanto, há uma transferência de renda dos produtores para a sociedade. Caso contrário, se $\mathrm{CPN}>1$, os produtores recebem valores superiores aos preços de mercado, logo, ocorre transferência de renda da sociedade para os produtores agrícolas.

d) Coeficiente de proteção efetiva (CPE) - informa a dimensão dos incentivos ou desestímulos que o sistema agrícola recebe das políticas sobre o produto e insumos comercializáveis. Este coeficiente demonstra os efeitos das transferências de políticas, responsáveis por tornar os preços do produto e dos insumos diferentes de seus valores de eficiência. Esses efeitos são expressos através da seguinte formulação matemática:

$\mathrm{CPE}=\frac{(\mathrm{A}-\mathrm{B})}{(\mathrm{E}-\mathrm{F})}$

Nota-se que, para o caso de $\mathrm{CPE}<1$, os produtores não possuem proteção quanto às políticas vigentes sobre o preço do produto e dos insumos comercializáveis, podendo estes sofrer os efeitos de taxações que tornam o valor adicionado à produção inferior ao valor adicionado em termos de eficiência. Por outro lado, se CPE $>1$, os produtores recebem proteção, ou seja, estão sendo beneficiados pelas transferências dessas políticas. A inexistência de transferência de políticas é verificada quando o CPE é igual a 1.

e) Coeficiente de lucratividade $(C L)$ - demonstra o efeito total das transferências de todas as políticas aplicadas ao sistema agrícola. Difere do CPN e do CPE, pois engloba no seu cálculo os incentivos das políticas sobre o mercado de fatores domésticos, sendo ilustrado pela equação:

$\mathrm{CL}=\frac{(\mathrm{A}-\mathrm{B}-\mathrm{C})}{(\mathrm{E}-\mathrm{F}-\mathrm{G})}$

Substituindo a Equação 1 e 2 no numerador e denominador, respectivamente, da Equação 8, o coeficiente de lucratividade, torna-se a razão entre o lucro privado e o lucro social, que pode ser reescrito da seguinte maneira:

$\mathrm{CL}=\frac{\mathrm{D}}{\mathrm{H}}$

f) Razão de subsídio ao produtor (RSP) - mostra a proporção de rendas, em termos de valores sociais, requerida para garantir a eficiência econômica do sistema agrícola analisado, caso "um único subsídio ou imposto substituísse todas as políticas de produtos, insumos e fatores, além das macroeconômicas" (Moss, 2006, p. 34; Sousa et al. 2011). Em síntese, indica a parcela de incentivo ou desincentivo integralizado ao sistema agrícola. Sendo, portanto, expresso pelas seguintes formulações:

$\mathrm{RSP}=\frac{(\mathrm{D}-\mathrm{H})}{\mathrm{E}} \quad$ ou $\quad \mathrm{RSP}=\frac{\mathrm{L}}{\mathrm{E}}$

Denota-se que valores de RSP $>1$ apontam para a presença de protecionismo. Quando o $\operatorname{RSP}<1$, não ocorre protecionismo no sistema agrícola, entretanto pode ocorrer a cobrança de subsídios. Para $\mathrm{RSP}=1$, o sistema se mantém em um cenário neutro, em que não ocorre nenhuma das situações anteriores. 


\subsection{Fonte de dados}

Os dados correspondentes aos custos de produção do consórcio cacaueiro e seringueira foram extraídos do trabalho do Virgens Filho (2003), correspondendo ao custo de preparo da área e plantio, tratos culturais, insumos e máquinas, implementos e equipamentos. Esse estudo técnico consiste no mais detalhado sobre o tema consorciação de cacaueiro e seringueira, o que justifica sua utilização neste estudo. Visando minimizar as discrepâncias nos valores, os dados foram corrigidos aplicando-se o Índice Geral de Preços (IGP-di) da Fundação Getúlio Vargas, a preços de janeiro de 2017.

O preço da arroba (@) ${ }^{1}$ de amêndoas de cacau foi obtido da Secretaria da Agricultura, Pecuária, Irrigação, Pesca e Aquicultura (Bahia, 2017), referente à média das cotações de janeiro de 2017 negociadas na "praça" de Ilhéus, resultando em R\$117,00/@. Já o preço do quilo do coágulo do látex com DRC ${ }^{2}$ (Dry Rubber Content) de 57\% extraído da seringueira foi obtido na Companhia Nacional de Abastecimento (2017), considerando o preço pago ao produtor na Bahia de $\mathrm{R} \$ 2,11 / \mathrm{Kg}$. Os preços sociais de cada produto foram levantados com base nos preços internacionais. Para o cacau, considerou-se o preço internacional disponibilizado pelo Banco Mundial de US $\$ 2,16 / \mathrm{Kg}$. Para a seringueira, considerou-se a média das cotações do látex com DRC de 60\%, no Conselho de Borracha da Malásia (Malaysian Rubber Board, 2017), equivalente a 710,70 RM Sen/Kg (Ringgit da Malásia). Foi aplicada uma taxa de câmbio de $\mathrm{R} \$ 3,196$, correspondente à média do mês de janeiro disponível no Banco Central do Brasil (2017), que resultou nos seguintes preços sociais: R\$103,55/@ de amêndoas de cacau e $\mathrm{R} \$ 5,17 / \mathrm{Kg}$ de coágulo de látex.

Para os fatores domésticos, mais precisamente o custo com mão de obra, equiparou-se ao salário médio vigente em território nacional ao salário médio praticado na Indonésia, utilizando um fator de conversão de aproximadamente $67 \%$ em relação ao custo da mão de obra no Brasil. A escolha deste país foi motivada pela sua representatividade tanto na cacauicultura como na heveicultura, ocupando nesta atividade a $3^{\mathrm{a}}$ e $2^{\mathrm{a}}$ posição no ranking de maiores produtores de cacau e de borracha natural, respectivamente (Food and Agriculture Organization of the United Nation, 2017).

Para os insumos comercializáveis de maior peso na planilha de custos, os preços estimados correspondem ao valor das exportações africanas e asiáticas para o Brasil, disponibilizados na base de dados AliceWeb do Ministério do Desenvolvimento, Indústria e Comércio Exterior e Serviços (Brasil, 2017), convertendo-os de US\$ FOB/Kg para R $\$ / \mathrm{Kg}$. Os demais produtos, ao considerar a ausência de divergências de políticas, seus preços sociais se tornam aproximações dos preços privados.

\section{RESULTADOS}

\subsection{MAP para sistemas produtivos no sul da Bahia}

Diante dos benefícios da consorciação de culturas, tem-se a possibilidade de avaliar a competitividade dos sistemas consorciados do cacau com a seringueira no sul da Bahia, sustentando-se nos indicadores do método da MAP. Nesse contexto, a Tabela 1 ilustra os valores obtidos na construção da MAP para esses sistemas produtivos, construídos a partir dos valores privados e sociais das receitas (preço multiplicado pela quantidade produzida), custos de produção (somatório dos insumos domésticos e comercializáveis) e lucros (diferença entre as receitas e os custos).

\footnotetext{
1 @=15 quilos.

${ }^{2}$ A referência do preço da borracha indica a quantidade de borracha seca (BS) contida no coágulo.
} 
Tabela 1 - Resultado da Matriz de Análise Política.

\begin{tabular}{ccccc} 
Discriminação & $\begin{array}{c}\text { Receitas } \\
\text { (R\$/ha) }\end{array}$ & \multicolumn{2}{c}{ Custos de Produção (R\$/ha) } & Lucros \\
\cline { 3 - 4 } & & $\begin{array}{c}\text { Insumos } \\
\text { comercializáveis }\end{array}$ & $\begin{array}{c}\text { Fatores } \\
\text { domésticos }\end{array}$ & (R/ha) \\
Preços privados & $\mathrm{A}$ & $\mathrm{B}$ & $\mathrm{C}$ & $\mathrm{D}$ \\
Preços sociais & $74.234,89$ & $25.529,32$ & $25.684,18$ & $23.021,39$ \\
Efeitos de divergência e & $\mathrm{E}$ & $\mathrm{F}$ & $\mathrm{G}$ & $\mathrm{H}$ \\
eficiência política & $95.157,64$ & $22.730,54$ & $16.951,56$ & $55.475,54$ \\
\hline
\end{tabular}

Fonte: Resultado da Pesquisa.

Primeiramente, observa-se que os valores sociais são maiores que os valores privados, em outras palavras, receitas obtidas na região sul da Bahia estão abaixo das receitas internacionais, resultando em valores negativos de transferências financeiras na magnitude de $28,17 \%$ para o consórcio. Considerando que o preço privado do cacau é superior ao seu preço social, infere afirmar que contribuiu para que a disparidade nas transferências financeiras no consórcio não fosse ainda maior. Em termos monetários, os montantes negativos de transferência associados à produção do consórcio foi equivalente a $\mathrm{R} \$-20.913,75$ para o período de 12 anos.

Observa-se que os custos dos insumos comercializáveis foi o que sofreu menores variações, ou seja, os valores privados foram superiores aos valores sociais em aproximadamente $12,31 \%$. Os custos dos fatores domésticos apresentaram valores privados superiores aos valores sociais, com variações iguais a 51,51\%. Entende-se que o custo com mão de obra é predominante na composição dos fatores domésticos, sendo a produção de amêndoas de cacau e de borracha natural intensiva em mão de obra. Foram desconsideradas nessa análise a utilização de mão de obra escrava, o descumprimento das leis trabalhistas ou contratos de trabalho e/ou quaisquer condições adversas que incidam sobre a remuneração salarial, devido à indisponibilidade dos dados e de mecanismos de estimação. Resultando no custo com mão de obra (fatores domésticos) mais elevado no Brasil do que internacionalmente.

A análise das variações entre o lucro social e privado segue uma interpretação diferente, uma vez que seu resultado está condicionado ao nível de receitas e de custos de produção. No entanto, a variação observada entre os resultados privados e sociais foram negativos para o consórcio, indicando que o lucro auferido por um produtor do sul da Bahia está abaixo do lucro auferido por um produtor no exterior.

\subsection{Análise dos indicadores privados e sociais}

Os resultados dos indicadores privados e sociais fornecidos pela matriz contábil para o sistema consorciado do cacaueiro com a seringueira é apresentado na Tabela 2. Salienta-se que a interpretação dos indicadores apresentados segue a metodologia adaptada por Oliveira et al. (2012) e utilizada por Pacheco (2014) para a formulação original de Monke \& Pearson (1989). Segundo os autores, essa releitura dos indicadores tem a finalidade de facilitar seu entendimento (por alguns deles se apresentarem em termos percentuais) e mensurar o desempenho e os impactos econômicos dos sistemas produtivos. 
Tabela 2 - Síntese dos indicadores da MAP para o consórcio cacaueiro-seringueira.

\begin{tabular}{|c|c|c|c|}
\hline Descrição do indicador & \multicolumn{2}{|c|}{ Dados da Fórmula } & Resultado \\
\hline \multirow{2}{*}{ Participação dos Lucros nas Receitas (PLR) } & Privado & $(D / A) * 100$ & 31,02 \\
\hline & Social & $(H / E) * 100$ & 58,30 \\
\hline \multirow{2}{*}{$\begin{array}{c}\text { Participação do Valor Adicionado nas } \\
\text { Receitas (PVAR) }\end{array}$} & Privado & $((A-B) / A)^{*} 100$ & 65,61 \\
\hline & Social & $((E-F) / E) * 100$ & 76,11 \\
\hline \multirow{2}{*}{$\begin{array}{c}\text { Participação dos Fatores Domésticos para o } \\
\text { Valor Adicionado (PFDVA) }\end{array}$} & Privado & $(C /(A-B)) * 100$ & 52,73 \\
\hline & Social & $(G /(E-F) * 100$ & 22,40 \\
\hline \multirow{2}{*}{ Produtividade Total dos Fatores (PTF) } & Privado & $A /(B+C)$ & 1,45 \\
\hline & Social & $\mathrm{E} /(\mathrm{F}+\mathrm{G})$ & 2,40 \\
\hline $\begin{array}{l}\text { Coeficiente de Proteção Nominal do Produto } \\
\text { (CPNP) }\end{array}$ & & $A / E$ & 0,78 \\
\hline $\begin{array}{l}\text { Coeficiente de Proteção Nominal dos } \\
\text { Insumos (CPNI) }\end{array}$ & & $B / F$ & 1,12 \\
\hline Coeficiente de Proteção Efetiva (CPE) & & $(\mathrm{A}-\mathrm{B}) /(\mathrm{E}-\mathrm{F})$ & 0,67 \\
\hline Vulnerabilidade da Cadeia às Políticas (VCP) & & $((H-D) / H)^{*} 100$ & 58,50 \\
\hline Coeficiente de Lucratividade $(\mathrm{CL})$ & & $\mathrm{D} / \mathrm{H}$ & 0,41 \\
\hline Nível de Tributação da Cadeia & & $(L / E) *(-1) * 100$ & 34,11 \\
\hline
\end{tabular}

Fonte: Resultados da pesquisa.

\subsubsection{Análise de Lucratividade}

A análise de lucratividade tem por finalidade avaliar a viabilidade econômica do sistema produtivo. Para atingir esse objetivo, utilizam-se três indicadores da MAP. São eles: Lucro Privado (LP), Lucro Social (LS) e Coeficiente de Lucratividade (CL).

Primeiramente, para analisar a eficiência dos sistemas produtivos, é necessário analisar a participação do lucro nas receitas ou resultados (PLR), no intuito de verificar a rentabilidade desses sistemas, e se eles são viáveis. Nesse contexto, observa-se que o consórcio apresentou lucro privado $(R \$ 23.021,39)$ e lucro social $(R \$ 55.475,55)$ positivos, resultando em PLRs positivas, equivalentes a $31,02 \%$, correspondendo a valores privados, e $58,30 \%$, a preços sociais.

Nesse contexto, a interpretação do CL possibilita mensurar numericamente a diferença entre os valores de LP e LS e mostra o montante de lucro social que foi transferido para os sistemas produtivos diante dessas falhas de políticas, ou nas palavras de Freitas (2013, p. 106), "o lucro que se obteria na ausência de políticas causadoras de distorção". O CL encontrado foi de 0,41 , demonstrando que o produtor não está sendo beneficiado com as políticas vigentes, ocorrendo taxações sobre os sistemas produtivos que reduzem diretamente o LP. Em síntese, os resultados do CL indicam a ausência de protecionismo no sistema produtivo.

A interpretação do $\mathrm{CL}$ em termos percentuais (1-CLx100) representa a vulnerabilidade do sistema produtivo em relação às políticas, representado pela sigla VCP. Com um CL de 0,41 , a interpretação desse indicador aponta que o produtor sofre uma taxação de $59 \%$ sobre a produção consorciada.

Desde os estudos pioneiros realizados pela Embrapa e por Vieira et al. (2001), a forte ausência de proteção às atividades agrícolas é evidenciada, estando presente, também, nos estudos mais recentes sobre as cadeias produtivas. No trabalho de Vieira et al. (2001), verificou-se que o menor CL entre as principais cadeias produtivas do Brasil, totalizando 11, incluindo a cacauicultura, atingiu $22 \%$. Os autores concluem que as políticas governamentais extraem recursos dos setores agrícolas, sendo, quase, impossível a eliminação destas distorções, uma vez que delas derivam a principal arrecadação sobre o setor: impostos e tributos. 


\subsubsection{Análise da Eficiência}

Para a análise de eficiência dos sistemas produtivos, foram considerados os indicadores Razão Custo Privado (RCP) e Razão Custo dos Insumos Domésticos (RCD), que indicam a participação dos fatores domésticos para o valor adicionado (PFDVA), sendo o primeiro utilizado a preços privados e o segundo a preços sociais.

Dada a característica da produção de cacau e de borracha natural, observa-se que, em comum, elas apresentam mão de obra intensiva, indicando o fator trabalho como totalidade na composição dos insumos domésticos. Isso justifica o fato de os custos com fatores domésticos no consórcio cacaueiro-seringueira serem superiores aos custos dos insumos comercializáveis. De acordo com Oliveira et al. (2012), as atividades intensivas em fatores domésticos (terra, capital e trabalho) estão mais expostas a sofrer prejuízos ocasionados por divergências de políticas, uma vez que essas atividades, de modo geral, apresentam baixa rentabilidade.

A partir dessa analogia, utiliza-se o indicado RCP para medir a capacidade do sistema de cobrir os custos dos fatores domésticos e manter-se competitivo.

Os índices de RCP, ou PFDVA privado (em termos percentuais) encontrado foi de 0,53 (52,73\%) para o consórcio. Assim, a partir desses resultados, entende-se que o sistema consorciado explorou melhor seus fatores domésticos. Por outro lado, o RCD, ou PFDVA social (em termos percentuais), sinaliza a eficiência relativa dos sistemas produtivos, medindo e comparando seus benefícios sociais. De acordo com Moss (2006, p. 47), este indicador segue o mesmo raciocínio do indicador de lucratividade privada, em que a minimização do RCD "é equivalente a maximizar os lucros sociais". Com isso, tem-se que o resultado igual a $0,22(22,4 \%)$, indicando a presença de eficiência econômica e vantagem competitiva a valores sociais. Com isso, tem-se que, para cada $R \$ 1,00$ economizado com a importação, seriam utilizados $\mathrm{R} \$ 0,22$ de recursos domésticos.

Em complementação a esta análise, utiliza-se o indicador de produtividade total dos fatores (PTF) para medir a razão entre o valor total da receita e o valor do custo total, obtido através da soma dos custos com fatores domésticos e o custo com insumos comercializáveis. De acordo com Araújo (2015, p. 85), o indicador de PTF, dentre os indicadores apresentados, capta melhor o nível de eficiência das atividades produtivas, permitindo a execução de análises comparativas entre elas, ou comparar mudanças em sua estrutura produtiva, a exemplo de "mudança na qualidade de um produto, melhoria do capital humano, tecnologia em todos os elos, financiamento adequado, pesquisa e desenvolvimento, entre outros". Em síntese, a PTF indica a taxa de retorno de investimentos dos fatores utilizados pelo sistema, e sua posição relativa em comparação com os demais.

Os resultados dos valores de PTF demonstraram que há uma relação positiva de eficiência e competitividade. Alternativamente, é possível interpretar esses indicadores da seguinte maneira: a receita é superior a $\mathrm{R} \$ 0,45$ aos custos com insumos comercializáveis e fatores domésticos para o sistema de produção consorciada. Já a receita a preços sociais supera os custos com insumos comercializáveis e fatores domésticos em $R \$ 1,40$.

\subsubsection{Análise de Proteção}

A presente seção visa analisar o nível de proteção dos sistemas produtivos, a partir da mensuração do nível de tributação e de subsídios que incidem sobre eles. São utilizados para atingir esse objetivo os seguintes indicadores: Coeficiente de Proteção Nominal do Produto (CPNP), Coeficiente de Proteção Nominal dos Insumos (CPNI) e o Coeficiente de Proteção Efetiva (CPE), cujos resultados são apresentados na Tabela 2.

O CPNP tem como função estimar o nível e o fluxo de tributações nos sistemas produtivos, ou seja, se há transferências de renda do sistema para a sociedade, via tributos, ou da sociedade para a atividade agrícola via impostos e/ou subsídios ao produtor. O resultado do CPNP encontrado para sistema consorciado foi igual a 0,78 , indicando uma desproteção dessa atividade, visto que seus preços estão abaixo dos preços internacionais.

Do ponto de vista da CPNI, que mede o grau de transferência ou o nível de proteção dos insumos comercializáveis, o resultado foi equivalente a 1,12. Diante disso, afirma-se que há 
transferência de renda dos produtores para a sociedade, pois os custos dos insumos comercializáveis foram aumentados por distorções de políticas.

Conforme mencionado por Ferreira Neto (2005) e por Moss (2006), os CPNP e o CPNI apresentam uma relação inversa, visto que o aumento dos preços privados para o produto afeta positivamente o nível de LP, enquanto o aumento dos preços privados dos insumos comercializáveis afeta negativamente o nível de LP. Refazendo as mesmas analogias propostas pelos autores, quanto menores os CPNPS e maiores os CPNIs, maiores as transferências dos produtores.

Apesar de configurar um importante indicador para medir a proteção das atividades produtivas, o CPN se limita apenas a comparações entre o preço privado e o preço social. Diante disso, Freitas (2013) afirma que a análise do CPE se torna mais eficiente para mensurar a proteção, pois avalia as transferências resultantes de políticas, tanto sobre o nível das receitas, quanto para os insumos comercializáveis, verificando, ainda, se tais políticas exercem estímulos ou desestímulos sobre os sistemas agrícolas.

O coeficiente calculado apresentou valores menores que a unidade, 0,67 , conforme apresentado por Oliveira et al. (2012), para CPEs menores que 1 (CPE <1), interpreta-se que a atividade produtiva está desprotegida e apresenta altas taxações, apresentando custos adicionais de $33 \%$. Corroborando os resultados apresentados anteriormente.

\subsubsection{Análise da Transferência de Políticas}

Para a construção da análise de transferência de políticas, foram utilizados os indicadores de Transferência Líquida de Política (TLP) e de razão de Subsídio ao Produtor (RSP), também denominado de Nível de Tributação da Cadeia (NTC).

O indicador TLP é resultante da diferença entre o LP e o LS, demonstrando os efeitos de divergências de políticas sobre a lucratividade do sistema produtivo. Como resultado desta análise, observou-se que o consórcio cacaueiro-seringueira apresentou um montante de R\$-32.454,15, por hectare, no total de 12 anos, demonstrando o quanto o produtor perde em decorrência das distorções de mercado e dos efeitos das políticas. Apesar da grande magnitude da diferença observada entre os valores privados, auferidos pelos produtores, e os valores sociais vigentes no mercado internacional, para Freitas (2013), não há indícios que apontem para uma relação direta entre o desequilíbrio de lucratividade e inviabilidade econômica, justificando-se através dos valores positivos de LPs verificados na sua aplicação da MAP para a produção leiteira. Compartilhando da visão do autor, a presente pesquisa, com base nos resultados do LP, confirma que as discrepâncias entre os preços sociais e privados não afetam a viabilidade econômica do sistema produtivo.

Para complementar a análise de transferências de políticas, foi mensurado o nível de tributação do consórcio com o intuito de demonstrar, numericamente, o volume de taxações e, consequentemente, a perda de rentabilidade. Para esta pesquisa, foi encontrado um RSP igual -0,34, cujos valores negativos expressam a presença de taxações. Em termos de taxação geral (NTC), os resultados foram de aproximadamente 34,11\%. O cálculo do RSP, ou NTC, engloba os efeitos de todas as políticas de tributação, impostos, encargos e juros sobre a rentabilidade das atividades produtivas, ou seja, demonstra, a preços sociais, o volume do produto ou receitas necessárias para garantir a eficiência econômica do sistema (Sousa et al., 2011). Quanto maior for o valor desses indicadores maior é a carga tributária. Esses resultados expressam o montante de desincentivos, em termos percentuais, gerados por falhas e distorções de políticas. Comparando com os resultados encontrados em outras pesquisas, tem-se taxações entre 18\% $(-0,18)$, identificados no cultivo da soja em 2001 (Alvim et al., 2004), e 64\% (-0,64), no cultivo da cana-de-açúcar em 2004 (Ferreira Neto, 2005).

\subsection{Análise de sensibilidade}

A análise de sensibilidade desempenha um importante papel no entendimento e na tomada de decisões por parte dos agentes, pois permite visualizar o comportamento das variáveis, dadas as mudanças no cenário, em outras palavras, analisa os efeitos de uma variação em uma variável isolada sobre as outras variáveis presentes no estudo. No contexto 
apresentado por esta pesquisa, a análise de sensibilidade tem como objetivo verificar a sensibilidade dos indicadores da MAP, dadas as variações na taxa de câmbio e nos preços sociais. Além disso, esta análise permite criar cenários diante de mudanças ou incertezas na estimação dos valores.

Nesta seção, são apresentados os efeitos de variações entre -10\% e 10\% na taxa de câmbio e nos preços sociais nos indicadores estimados para o consórcio cacaueiro-seringueira (Tabela 3), apresentados na seção anterior. Para tanto, foi utilizado o método de Monte Carlo, através do software @RISK que permite analisar os efeitos de 1.000 interações, dadas as variáveis de entrada (taxa de câmbio e preços sociais) e as variáveis de saída (os indicadores da MAP).

Tabela 3 - Estatísticas de sensibilidade dos indicadores da MAP para o sistema de consórcio cacaueiro e seringueira, dadas as variações entre $-10 \%$ e $10 \%$ na taxa de câmbio.

\begin{tabular}{|c|c|c|c|c|c|c|c|c|c|}
\hline \multirow{2}{*}{ ESTATÍSTICAS } & \multicolumn{9}{|c|}{ VARIÁVEIS DE SAIIDA } \\
\hline & LP & LS & TLP & $\mathrm{RCP}$ & RCD & CPN & CPE & $\mathrm{CL}$ & RSP \\
\hline Mínimo & $23.021,39$ & $49.061,09$ & $-38.866,55$ & 0,53 & 0,23 & 0,71 & 0,61 & 0,37 & $-0,37$ \\
\hline Máximo & $23.021,39$ & $61.887,95$ & $-26.039,70$ & 0,53 & 0,24 & 0,87 & 0,76 & 0,47 & $-0,30$ \\
\hline Média & $23.021,39$ & $55.475,47$ & $-32.454,08$ & 0,53 & 0,23 & 0,78 & 0,67 & 0,42 & $-0,34$ \\
\hline Moda & $23.021,39$ & $55.310,11$ & $-32.288,72$ & 0,53 & 0,23 & 0,78 & 0,67 & 0,42 & $-0,34$ \\
\hline Desvio Padrão & 0,00 & $2.667,03$ & $2.667,03$ & 0,00 & 0,00 & 0,03 & 0,03 & 0,02 & 0,01 \\
\hline \multicolumn{10}{|c|}{ PERCENTIS } \\
\hline $5 \%$ & $23.021,39$ & $51.002,22$ & $-36.922,51$ & 0,53 & 0,23 & 0,73 & 0,62 & 0,38 & $-0,36$ \\
\hline $10 \%$ & $23.021,39$ & $51.860,16$ & $-36.064,01$ & 0,53 & 0,23 & 0,74 & 0,63 & 0,39 & $-0,36$ \\
\hline $15 \%$ & $23.021,39$ & $52.512,09$ & $-35.413,17$ & 0,53 & 0,23 & 0,75 & 0,64 & 0,39 & $-0,36$ \\
\hline $20 \%$ & $23.021,39$ & $53.070,78$ & $-34.859,19$ & 0,53 & 0,23 & 0,75 & 0,65 & 0,40 & $-0,35$ \\
\hline $25 \%$ & $23.021,39$ & $53.557,76$ & $-34.372,70$ & 0,53 & 0,23 & 0,76 & 0,65 & 0,40 & $-0,35$ \\
\hline $30 \%$ & $23.021,39$ & $53.999,86$ & $-33.931,23$ & 0,53 & 0,23 & 0,76 & 0,66 & 0,40 & $-0,35$ \\
\hline $35 \%$ & $23.021,39$ & $54.404,92$ & $-33.526,51$ & 0,53 & 0,23 & 0,77 & 0,66 & 0,41 & $-0,35$ \\
\hline $40 \%$ & $23.021,39$ & $54.779,86$ & $-33.146,97$ & 0,53 & 0,23 & 0,77 & 0,66 & 0,41 & $-0,34$ \\
\hline $45 \%$ & $23.021,39$ & $55.136,26$ & $-32.790,50$ & 0,53 & 0,23 & 0,78 & 0,67 & 0,41 & $-0,34$ \\
\hline $50 \%$ & $23.021,39$ & $55.469,78$ & $-32.456,37$ & 0,53 & 0,23 & 0,78 & 0,67 & 0,41 & $-0,34$ \\
\hline $55 \%$ & $23.021,39$ & $55.808,28$ & $-32.121,02$ & 0,53 & 0,23 & 0,78 & 0,68 & 0,42 & $-0,34$ \\
\hline $60 \%$ & $23.021,39$ & $56.162,96$ & $-31.765,97$ & 0,53 & 0,23 & 0,79 & 0,68 & 0,42 & $-0,34$ \\
\hline $65 \%$ & $23.021,39$ & $56.541,36$ & $-31.391,38$ & 0,53 & 0,23 & 0,79 & 0,69 & 0,42 & $-0,34$ \\
\hline $70 \%$ & $23.021,39$ & $56.941,45$ & $-30.984,48$ & 0,53 & 0,23 & 0,80 & 0,69 & 0,43 & $-0,33$ \\
\hline $75 \%$ & $23.021,39$ & $57.381,89$ & $-30.549,60$ & 0,53 & 0,24 & 0,80 & 0,70 & 0,43 & $-0,33$ \\
\hline $80 \%$ & $23.021,39$ & $57.870,27$ & $-30.060,48$ & 0,53 & 0,24 & 0,81 & 0,70 & 0,43 & $-0,33$ \\
\hline $85 \%$ & $23.021,39$ & $58.421,50$ & $-29.501,45$ & 0,53 & 0,24 & 0,82 & 0,71 & 0,44 & $-0,32$ \\
\hline $90 \%$ & $23.021,39$ & $59.077,11$ & $-28.848,88$ & 0,53 & 0,24 & 0,83 & 0,72 & 0,44 & $-0,32$ \\
\hline $95 \%$ & $23.021,39$ & $59.930,48$ & $-27.999,53$ & 0,53 & 0,24 & 0,84 & 0,73 & 0,45 & $-0,32$ \\
\hline
\end{tabular}

Fonte: Resultado da pesquisa. 
Analisando os efeitos que a taxa de câmbio exerce sob o sistema de produção consorciada do cacaueiro com a seringueira, observa-se que o LS varia entre R\$49.061,09 e $\mathrm{R} \$ 61.887,95$, dadas as variações $-10 \%$ e $10 \%$ na taxa de câmbio. A mesma magnitude entre os valores mínimo e máximo do LS é encontrada para a TLP, visto que, assim como nos modelos anteriores, o LP não é influenciado diretamente pela taxa de câmbio, portanto apresenta-se constante na análise se sensibilidade.

Considerando o comportamento dos demais indicadores, verificou-se que o $70 \%$ das interações do RCD foram iguais a 0,23, sendo o restante igual a 0,24. Para o CPN, os resultados variaram entre 0,71 e 0,87. Para o CPE, essas variações encontram-se entre 0,61 e 0,76. Em ambos os casos, verificou-se que as mudanças de cenários da taxa de câmbio não geram indicadores maiores que a unidade (1), para o sistema de produção do consorciado. Considerando o $\mathrm{CL}$, os resultados mínimo e máximo foram, respectivamente, 0,37 e 0,47, sendo que apenas 5\% das interações realizadas estavam abaixo de 0,38, e 5\% acima de 0,45. Por fim, o RSP variou entre $-0,37$ e $-0,30$, sendo $10 \%$ dos valores menores que $-0,10$, e $10 \%$ maiores que $-0,09$.

Na Tabela 4, são apresentados os efeitos das variações de $-10 \%$ a $10 \%$ nos valores sociais sobre os indicadores mensurados para o sistema de produção consorciada do cacaueiro com a seringueira.

Tabela 4 - Estatísticas de sensibilidade dos indicadores da MAP para o sistema de consórcio cacaueiro e seringueira, dadas as variações entre $-10 \%$ e $10 \%$ nas receitas e custos, a preços sociais.

\begin{tabular}{|c|c|c|c|c|c|c|c|c|c|}
\hline \multirow{2}{*}{ ESTATÍSTICAS } & \multicolumn{9}{|c|}{ VARIÁVEIS DE SAÍDA } \\
\hline & LP & LS & TLP & $\mathbf{R C P}$ & RCD & CPN & CPE & CL & RSP \\
\hline Mínimo & $23.021,39$ & $44.368,40$ & $-42.143,50$ & 0,53 & 0,19 & 0,71 & 0,59 & 0,35 & $-0,41$ \\
\hline Máximo & $23.021,39$ & $65.164,89$ & $-21.347,01$ & 0,53 & 0,29 & 0,86 & 0,78 & 0,52 & $-0,25$ \\
\hline Média & $23.021,39$ & $55.475,43$ & $-32.454,04$ & 0,53 & 0,23 & 0,78 & 0,67 & 0,42 & $-0,34$ \\
\hline Moda & $23.021,39$ & $54.593,20$ & $-31.571,81$ & 0,53 & 0,23 & 0,78 & 0,69 & 0,42 & $-0,34$ \\
\hline Desvio Padrão & 0,00 & $4.003,91$ & $4.003,91$ & 0,00 & 0,02 & 0,03 & 0,04 & 0,03 & 0,03 \\
\hline \multicolumn{10}{|c|}{ PERCENTIS } \\
\hline $5 \%$ & $23.021,39$ & $48.878,51$ & $-39.084,91$ & 0,53 & 0,21 & 0,73 & 0,62 & 0,37 & $-0,39$ \\
\hline $10 \%$ & $23.021,39$ & $50.282,22$ & $-37.988,69$ & 0,53 & 0,21 & 0,74 & 0,62 & 0,38 & $-0,38$ \\
\hline $15 \%$ & $23.021,39$ & $51.145,55$ & $-36.823,20$ & 0,53 & 0,22 & 0,75 & 0,63 & 0,38 & $-0,37$ \\
\hline $20 \%$ & $23.021,39$ & $51.879,11$ & $-36.096,29$ & 0,53 & 0,22 & 0,75 & 0,64 & 0,39 & $-0,37$ \\
\hline $25 \%$ & $23.021,39$ & $52.572,33$ & $-35.407,14$ & 0,53 & 0,22 & 0,76 & 0,65 & 0,39 & $-0,36$ \\
\hline $30 \%$ & $23.021,39$ & $53.123,97$ & $-34.720,66$ & 0,53 & 0,23 & 0,76 & 0,65 & 0,40 & $-0,36$ \\
\hline $35 \%$ & $23.021,39$ & $53.738,35$ & $-34.018,80$ & 0,53 & 0,23 & 0,77 & 0,66 & 0,40 & $-0,35$ \\
\hline $40 \%$ & $23.021,39$ & $54.350,51$ & $-33.442,60$ & 0,53 & 0,23 & 0,77 & 0,66 & 0,41 & $-0,35$ \\
\hline $45 \%$ & $23.021,39$ & $54.788,71$ & $-32.968,19$ & 0,53 & 0,23 & 0,78 & 0,67 & 0,41 & $-0,34$ \\
\hline $50 \%$ & $23.021,39$ & $55.348,42$ & $-32.347,60$ & 0,53 & 0,23 & 0,78 & 0,67 & 0,42 & $-0,34$ \\
\hline $55 \%$ & $23.021,39$ & $55.956,59$ & $-31.785,82$ & 0,53 & 0,24 & 0,78 & 0,68 & 0,42 & $-0,34$ \\
\hline $60 \%$ & $23.021,39$ & $56.457,05$ & $-31.330,84$ & 0,53 & 0,24 & 0,79 & 0,68 & 0,42 & $-0,33$ \\
\hline $65 \%$ & $23.021,39$ & $57.025,13$ & $-30.733,81$ & 0,53 & 0,24 & 0,79 & 0,69 & 0,43 & $-0,33$ \\
\hline $70 \%$ & $23.021,39$ & $57.680,49$ & $-30.107,17$ & 0,53 & 0,24 & 0,80 & 0,69 & 0,43 & $-0,32$ \\
\hline $75 \%$ & $23.021,39$ & $58.421,84$ & $-29.567,59$ & 0,53 & 0,25 & 0,80 & 0,70 & 0,44 & $-0,32$ \\
\hline $80 \%$ & $23.021,39$ & $59.057,55$ & $-28.872,05$ & 0,53 & 0,25 & 0,81 & 0,71 & 0,44 & $-0,31$ \\
\hline $85 \%$ & $23.021,39$ & $59.839,70$ & $-28.175,13$ & 0,53 & 0,25 & 0,82 & 0,72 & 0,45 & $-0,31$ \\
\hline $90 \%$ & $23.021,39$ & $60.990,61$ & $-27.300,89$ & 0,53 & 0,26 & 0,83 & 0,72 & 0,46 & $-0,30$ \\
\hline $95 \%$ & $23.021,39$ & $62.097,01$ & $-25.872,09$ & 0,53 & 0,26 & 0,84 & 0,74 & 0,47 & $-0,29$ \\
\hline
\end{tabular}

Fonte: Resultado da pesquisa. 
Observa-se, primeiramente, que as mudanças nos preços sociais não afetam os indicadores privados LP e RCP, fazendo com que esses valores não se alterem. Por outro lado, os indicadores sociais são sensíveis a estas variações. Neste caso, tem-se como exemplo o LS que apresentou valores mínimo e máximo respectivamente iguais a $R \$ 44.368,40$ e $R \$ 65.164,89$, enquanto o TLP variou entre $R \$-42.143,50$ e $R \$-21.347,01$. Apenas $5 \%$ das interações apresentaram LS menores que $\mathrm{R} \$ 48.878,51$ e $5 \%$ foram maiores que $\mathrm{R} \$ 62.097,01$.

Considerando o comportamento dos demais indicadores, verificou-se que o RCD apresentou as menores variações, visto que os valores mínimo e máximo foram, respectivamente, 0,19 e 0,29, sendo que 15\% dos valores encontrados foram iguais a 0,22, mesmo valor encontrado no cálculo da MAP. Para o CPN, os resultados variaram entre 0,71 e 0,86. Para o CPE, essas variações encontram-se entre 0,59 e 0,78. Considerando o CL, os resultados mínimo e máximo foram, respectivamente, 0,35 e 0,52, sendo que apenas 20\% das interações realizadas estavam abaixo de 0,39, e 15\% acima de 0,45. Por fim, o RSP variou entre -0,41 e -0,25, sendo o valor médio igual a -0,34, assim como no cálculo original para esse sistema produtivo.

Em síntese, a Tabela 5 ilustra o grau de sensibilidade dos indicadores da MAP, em resposta às variações de $10 \%$ na taxa de câmbio e nos valores sociais.

Tabela 5 - Sensibilidade dos indicadores da MAP para o consórcio cacaueiro-seringueira, dadas as variações de $10 \%$ na taxa de câmbio e nos valores sociais.

\begin{tabular}{ccccc} 
& Taxa de & \multicolumn{3}{c}{ Valores Sociais } \\
\cline { 3 - 5 } Indicadores & 0,12 & Receitas & $\begin{array}{c}\text { Insumos } \\
\text { comercializáveis }\end{array}$ & $\begin{array}{c}\text { Fatores } \\
\text { domésticos }\end{array}$ \\
\cline { 3 - 5 } LS & 0,20 & 0,17 & $-0,04$ & $-0,03$ \\
TLP & 0,29 & $-0,07$ & $-0,05$ \\
RCD & $-0 ., 01$ & $-0,12$ & 0,03 & 0,10 \\
CPN & $-0,09$ & $-0,09$ & - & - \\
CPE & $-0,10$ & $-0,12$ & 0,03 & - \\
CL & $-0,10$ & $-0,15$ & 0,04 & 0,03 \\
RSP & 0,09 & 0,18 & $-0,07$ & $-0,05$
\end{tabular}

Fonte: Resultados da pesquisa.

O aumento de $10 \%$ na taxa de câmbio e nas receitas a preço social eleva o lucro social em $12 \%$ e $17 \%$, respectivamente. Enquanto o aumento de $10 \%$ nos custos com insumos comercializáveis reduz o LS em $4 \%$ e com fatores domésticos reduz em 3\%. De modo semelhante ocorre com o TLP, que aumenta em $20 \%$ e $29 \%$, dada a elevação da taxa de câmbio e das receitas sociais, e reduz 7\% e 5\%, com a elevação dos custos com insumos comercializáveis e fatores domésticos, respectivamente.

O aumento de 10\% na taxa de juros reduz em níveis de 1\%, 9\%, 10\% e 10\% os indicadores $\mathrm{RCD}, \mathrm{CPN}, \mathrm{CPE}$ e $\mathrm{CL}$, respectivamente. Além disso, os incrementos nas receitas, mantido tudo mais constante, reduz o RCD, CPN CPE e CL em 12\%, 9\%, 12\% e 15\%.

O CPN não é sensível às variações dos custos sociais. E o CPE não varia devido às mudanças nos fatores domésticos. A justificativa para essas duas situações é que esses componentes não fazem parte do cálculo desses indicadores.

De modo geral, os indicadores da MAP se mostraram mais sensíveis às mudanças nas receitas sociais do que às variações da taxa de câmbio e dos custos de produção. Indica-se, portanto, que medidas que estimulem o aumento das receitas, tanto através de estímulos à produção quanto aos preços, tendem a beneficiar o comportamento dos indicadores da MAP. Todavia, medidas que visem à redução dos custos de produção são importantes para garantir a eficiência do sistema produtivo e aumentar a margem de lucro. 


\section{CONSIDERAÇÕES FINAIS}

O consórcio cacaueiro-seringueira se mostrou eficiente e competitivo, através da demonstração dos resultados dos indicadores da MAP. De modo geral, observou-se que o produtor sofre penalizações em decorrência de políticas taxativas e de tributação, ocorrendo, portanto, uma transferência de renda do produtor à sociedade. Todavia, a produção consorciada do cacau com a seringueira representa uma atividade atrativa, do ponto de vista competitivo, dados os valores das receitas e custos de produção, e apresenta vantagem comparativa, dados os preços internacionais (sociais). Em outras palavras, a análise infere que, apesar de apresentar resultados que a caracterizam como uma atividade lucrativa, o consórcio sofre uma desproteção por parte das políticas governamentais, neste caso representadas por impostos e tributos, as taxas de juros e as taxas de câmbio e por políticas comerciais sob a produção e a comercialização.

O consórcio apresentou resultados favoráveis, indicando que este representa uma atividade potencial economicamente. Ressalva-se que estes resultados são dependentes das oscilações dos preços, muito comuns no mercado internacional de cacau. Em outras palavras, a conjuntura de preços, tanto para o cacau quanto para a borracha natural, interferem nos resultados dos indicadores da MAP.

Verifica-se uma otimização dos recursos, comercializáveis e domésticos, uma vez que os custos de produção do consórcio não superam o somatório dos custos com a cacauicultura e a heveicultura. Esse resultado confirma as principais vantagens da produção consorciada, relacionadas ao maior aproveitamento do solo e dos insumos produtivos, e o aumento da produtividade e do lucro em decorrência da produção simultânea de duas culturas. Todavia, atenta-se que a limitação de dados de custos de produção da heveicultura faz com que o desempenho da produção consorciada fique condicionada ao desempenho da produção de cacau. Em outras palavras, o cacau se torna a cultura predominante dentro do consórcio produtivo, fazendo com que os valores reais fiquem camuflados, problema que pode ser corrigido ao levantar dados de custos para sistemas com mais de 20 anos.

Em termos metodológicos, a pesquisa reafirma a eficiência da Matriz de Análise de Política para comparar as distorções que afetam diferentes sistemas produtivos e os impactos que estas exercem sobre os níveis de lucratividade e eficiência. Além disso, possibilita mensurar o nível de proteção que as medidas políticas, incidentes sobre o produto e sobre os insumos, podem exercer na forma de ganhos e perdas para o produtor.

No âmbito das políticas públicas, as altas taxas de impostos e tributações que incidem sobre os insumos comercializáveis e fatores domésticos contribuem para a perda de competitividade dos sistemas produtivos, visto que o alto custo de produção é um dos principais entraves que penalizam o produtor na região, refletindo uma realidade do contexto brasileiro. Dentre as medidas que podem ser implementadas para melhorar a competitividade da cacauicultura e da heveicultura no sul da Bahia, destacam-se: a redução da carga tributária incidente sobre os insumos e fatores de produção, e sobre o preço do produto; maior investimento em pesquisa e desenvolvimento (P\&D) que visam o aumento da qualidade do produto, da produtividade e da eficiência na alocação dos recursos; ampliação das políticas de financiamento e concessão de crédito agrícola aos pequenos e médios produtores; maior controle dos mecanismos comerciais, tais como taxas de exportação e importação, subsídios, restrições e outras políticas comerciais; e a manutenção de uma política macroeconômico compatível com os interesses de investimentos, incluindo, portanto, o controle inflacionário, manutenção de taxas de juros favoráveis e uma política cambial que estimule as exportações, dando condições de competir no mercado internacional.

Como sugestões para estudos futuros, destaca-se a necessidade do constante monitoramento das atividades economicamente representativas para a região sul da Bahia, como a cacauicultura e a heveicultura. Ainda assim, para outras culturas potenciais do Estado, como a soja, o algodão e o setor frutífero, resultando em uma atualização da literatura existente e ampliação das pesquisas que utilizam o método MAP para a Bahia e também para o Brasil. Salienta-se que estudos que visam analisar a competitividade e as fragilidades em atividades produtivas, sobretudo nas atividades ligadas ao setor primário agrícola e ao setor agroindustrial, apresentam grande relevância para a elaboração de políticas públicas 
eficientes, ao identificar as lacunas que impedem seu desenvolvimento e propor soluções direcionadas, além de beneficiar produtores e a economia regional como um todo.

\section{AGRADECIMENTOS}

À Coordenação de Aperfeiçoamento de Pessoal de Nível Superior (CAPES) pelo financiamento concedido.

\section{REFERÊNCIAS}

Adesina, A. A., \& Coulibaly, O. N. (1998). Policy and competitiveness of agroforestry-based technologies for maize production in Cameroon: an application of policy analysis matrix. Agricultural Economics, 19(1-2), 1-13.

Alvim, M. I. S. A., Valle, S. M. L. R., Lima, J. E., \& Silva, O. M. (2004). Análise da competitividade da produção de soja nos sistemas de plantio direto e plantio convencional na região do cerrado brasileiro. Revista de Economia e Sociologia Rural, 42(2), 223-242.

Araújo, W. B. C. (2015). Matriz de Análise Política aplicada à cadeia produtiva da manga no município de Petrolina, Pernambuco (Dissertação de mestrado). Programa de Pós-graduação em Economia Rural, Universidade Federal do Ceará, Fortaleza.

Bahia. Secretaria da Agricultura, Pecuária, Irrigação, Pesca e Aquicultura - SEAGRI. (2017). Cotações agrícolas. Recuperado em 22 de abril de 2017, de http://www.seagri.ba.gov.br/cotacao?produto=512\&praca=286176\&tipo=368\&data_inicio=01\%2F04 \%2F2017\&data_final=22\%2F04\%2F2017

Banco Central do Brasil - BACEN. (2017). Cotações e boletins. Recuperado em 8 de setembro de 2017, de http://www4.bcb.gov.br/pec/taxas/port/ptaxnpesq.asp?id=txcotacao

Brasil. Ministério do Desenvolvimento, Indústria e Comércio - MDIC. (2017). Aliceweb. Recuperado em 20 de abril de 2017, de http://aliceweb.mdic.gov.br/

Companhia Nacional de Abastecimento - CONAB. (2017). Preços agropecuários. Recuperado em 23 de abril de 2017, de http://www.conab.gov.br/detalhe.php?a=1409\&t=2

Fang, C., \& Beghin, J. C. (2000). Food self-sufficiency, comparative advantage, and agricultural trade: a policy Analysis Matrix for chinese agriculture (Working Paper, No. 99-WP 223). Ames: Center for Agricultural and Rural Development and Department of Economics.

Ferreira Neto, J. (2005). Competitividade da produção de cana-de-açúcar no Brasil (Dissertação de mestrado). Programa de Pós-graduação em Economia Aplicada, Universidade Federal de Viçosa, Minas Gerais, 2005.

Food and Agriculture Organization of the United Nation - FAO. (2017). Faostat. Rome. Recuperado em 29 de março de 2017, de http://www.fao.org/faostat/en/\#data/QC

Freitas, J. B. (2013). Competitividade, eficiência econômica e efeitos de políticas em diferentes níveis tecnológicos na cadeia produtiva do leite em pó integral no Rio grande do Sul: uma análise do método da Matriz de Análise Política (MAP) (Dissertação de mestrado). Programa de Pós-graduação em Agronegócios, Universidade Federal do Rio Grande do Sul, Porto Alegre.

Kanaka, S., \& Chinnadurai, M. (2015). The policy analysis matrix of rice cultivation in India. European Journal of Basic and Applied Sciences, 2(1), 8-19.

Malaysian Rubber Board. (2017). Malaysian Rubber Exchange. Recuperado em 15 de setembro de 2017, de http://www3.Igm.gov.my/mre/MonthlyPrices.aspx

Milhomem, A. V., \& Teixeira, S. M. (2001). Competitividade dos sistemas produtivos de café no Brasil. In Anais do $2^{\circ}$ Simpósio de Pesquisa dos Cafés do Brasil (pp. 2136-2144) Vitória: Centro de Convenções de Vitória.

Mohanty, S., Fan, C., \& Chaudhary, J. (2002). Assessing the competitiveness of Indian cotton production: a policy analysis matrix approach (Working Paper, No. 02-WP 301). Ames: Center for Agricultural and Rural Development and Department of Economics.

Monke, A. E., \& Pearson, S. R. (1989). The policy analysis matrix for agricultural development. New York: Cornell University Press.

Moss, S. R. (2006). Competitividade da produção do café arábica em Minas Gerais e São Paulo (Dissertação de mestrado). Programa de Pós-graduação em Economia Aplicada, Universidade Federal de Viçosa, Viçosa. 
Oliveira, A. J., Torres, D. A. P., Talamini, D. J. D., Martins, F. M., Lima Filho, J. R., Belarmino, L. C., \& Lopes, M. R. (2012). Matriz de análise política: metodologia e análise. Brasília: Embrapa.

Pacheco, M. T. M. (2014). Análise do desempenho na cadeia produtiva da maçã no corredor Vacaria - RS ao Porto de Rio Grande-RS: sob a ótica da Matriz de Análise de Política (MAP) (Dissertação de mestrado). Programa de Pós-graduação em Administração, Universidade de Caxias do Sul, Caxias do Sul.

Pearson, S., Gotsch, C., \& Bahri, S. (2003). Aplications of the policy analysis matrix in Indonesian agriculture. Indonesia: Yayasan Obor Indonesia.

Pires, M. M. (1996). Perspectivas de expansão da produção de grãos em Minas Gerais no contexto da liberalização dos mercados (Dissertação de mestrado). Programa de Pós-graduação em Economia. Universidade Federal de Viçosa, Viçosa.

Rosado, P. L., Tosto, S. G., \& Gomes, M. F. M. (2006). Competitividade e expansão da produção de borracha natural brasileira, no contexto de liberalização dos mercados. In A. P. Alvarenga, P. L. Rosado, C. A. F. S. Carmo \& S. G. Tôsto (Eds.), Seringueira: aspectos econômicos sociais e perspectivas para o seu fortalecimento (pp. 103-128). Viçosa: EPAMIG, EMBRAPA.

Soares, N. S., Silva, M. L., Rezende, J. L. P., \& Gomes, M. F. M. (2010). Competitividade da cadeia produtiva da madeira de eucalipto no Brasil. Revista Árvore, 34(5), 917-928.

Sousa, G. S., Pires, M. M., \& Rosado, P. L. (2011). Efeitos de políticas sobre a competitividade e eficiência da cadeia produtiva de biodiesel de dendê na região do Baixo Sul da Bahia. Revista Economica do Nordeste, 42(4), 827-841.

Superintendência de Estudos Econômicos e Sociais da Bahia - SEl. (2017). Sistema de Informações Municipais. Recuperado em 29 de novemro de 2017, de http://sim.sei.ba.gov.br/sim/tabelas.wsp

Vieira, R. C. M. T., Teixeira Filho, A. R., Oliveira, A. J., \& Lopes, M. R. (2001). Cadeias produtivas no Brasil: análise da competitividade. Revista de Política Agrícola, 10(4), 7-15.

Virgens Filho, A. C. (2003). Programa de desenvolvimento do agronegócio da borracha no Estado da BahiaPRODEAB. Ilhéus: CEPLAC. $119 \mathrm{p}$.

\author{
Submetido: 21/Fev./2018 \\ Aceito: 28/Jun./2019 \\ Classificação JEL: Government Policy and Regulation
}

